\title{
Inhibition of Eye Movements Disrupts Spatial Sequence Learning
}

\author{
Srdan Medimorec ${ }^{1}$, Petar Milin ${ }^{2}$ and Dagmar Divjak ${ }^{2,3}$ \\ ${ }^{1}$ Department of Psychology, Teesside University \\ ${ }^{2}$ Department of Modern Languages, University of Birmingham \\ ${ }^{3}$ Department of English Language and Linguistics, University of Birmingham
}

\begin{abstract}
Author Note
Correspondence concerning this paper should be addressed to: Srdan Medimorec, Department of Psychology, Teesside University, Middlesbrough, Tees Valley, TS1 3BX, UK. Email: s.medimorec@tees.ac.uk
\end{abstract}


PREDICTION AND IMPLICIT SEQUENCE LEARNING

\begin{abstract}
Implicit sequence learning is an integral part of human experience, yet the nature of the mechanisms underlying this type of learning remains a matter of debate. In the current study we provide a test for two accounts of implicit sequence learning, i.e., one that highlights sequence learning in the absence of any motor responses (with suppressed eye-movements) and one that highlights the relative contribution of the motor processes (i.e., eye movements) to learning. To adjudicate between these accounts and determine whether a motor response is a requisite process in sequence learning, we used anticipation measures to compare performance on the standard oculomotor serial reaction time (SRT) task and on a version of the SRT task where the eyemovements were restricted during the learning phase. Consistent with the response-based account, our results demonstrated an increased proportion of correct anticipations in the standard SRT task compared to the restricted-movement task.
\end{abstract}

Keywords: implicit learning, sequence learning, motor learning, perceptual learning, SRT task 


\section{PREDICTION AND IMPLICIT SEQUENCE LEARNING}

Sequence learning is one of the remarkable cognitive capabilities underlying different aspects of human behavior, ranging from language to various motor skills (Cleeremans \& McClelland, 1991; Nissen \& Bullemer, 1987; Stadler, 1989; Saffran, Aslin, \& Newport, 1996). A large amount of evidence indicates that structured sequences can be acquired implicitly, without awareness and without explicit instruction (Nissen \& Bullemer, 1987; Stadler \& Frensch 1998; Turk-Browne, Scholl, Chun, \& Johnson, 2009). However, the exact nature of the cognitive mechanism underlying implicit sequence learning remains a matter of debate (e.g., Schwarb \& Schumacher, 2012). The relative contribution of the motor and perceptual processes to implicit sequence learning remains unclear, as various learning tasks used to elicit implicit learning usually involve a combination of both (i.e., there is usually a motor response to perceived stimuli). Importantly, understanding the mechanism behind implicit sequence learning provides an opportunity to gain a deeper understanding of learning in general. In the current study we provide a test for different potential theoretical accounts of implicit learning.

\section{Implicit Sequence Learning}

A commonly used method to study implicit learning is the serial reaction time task (SRT task; Nissen \& Bullemer, 1987). Typically, in this task individuals are presented with a rapid sequence of elements that appear on a screen and asked to press keys corresponding to the target location on the screen (upon detection or predictively). Critically, unknown to the participants the stimuli presentation follows a fixed (i.e., to-be-learned) sequence, and at some point, a different (i.e., interfering) sequence is inserted, followed by another presentation of the original sequence (for a review, see Schwarb \& Schumacher, 2012). Learning is assessed using different measures, including reduced RT across the sequence presentation, or increased RT when switching to a different sequence. Another typical way of assessing learning involves contrasting 


\section{PREDICTION AND IMPLICIT SEQUENCE LEARNING}

the interfering block with the test block at the end (the results of such an analysis are sometimes argued to account for the motor component, even though in this way, the motor component is controlled at test only and not during learning). After the task, participants' sequence awareness is assessed. If participants cannot verbalize or recall the sequence, it is argued that knowledge is implicit. Clearly, the described implementation of the paradigm involves a combination of perceptual and motor involvement, leaving the specific nature of the learning somewhat unclear. An unresolved question about the nature of learning in the SRT task is whether a motor response is a requisite process in sequence learning.

According to one account, sequential learning is realized through a formation of stimulus-stimulus associations, while motor responses are not necessary for learning (Cohen, Ivry, \& Keele, 1990; Howard, Mutter, \& Howard, 1992). For example, Howard et al. (1992) asked groups of participants to perform the standard SRT task by responding with keypresses to a repeating spatial pattern, or to simply observe the pattern (after making manual responses to the initial pattern only). Learning was tested by introducing an interfering transfer block. The study demonstrated that individuals learned a sequence even when they did not make responses (i.e., when their responses were reduced to the initial pattern only). However, it has been argued that since participants in the experiment were provided with the short initial manual practice, the observed learning should not be considered purely perceptual (e.g., Marcus et al., 2006). Moreover, since watching a sequence on a screen involved eye movements, there is a possibility that the oculomotor responses supported sequence learning (Willingham, 1999). More recent research has examined the role of eye movements in perceptual sequence learning (Coomans, Deroost, Voandenbossche, Van der Busche, \& Soetens, 2012; see also Haider, Eberhardt, Kunde, \& Rose, 2013; Remillard, 2011). For example, Coomans et al. (2012) asked participants to fixate 


\section{PREDICTION AND IMPLICIT SEQUENCE LEARNING}

on a cross in the middle of a screen, and react manually to targets (letter pairs) around the cross ("C $\mathrm{C}$ " response for the letter pair "XO", "N" for the pair " $\mathrm{OX}$ "). Thus, participants responded to target identities, rather than positions (since the spatial position of target presentation was not relevant for responding). Learning in this study referred to a task-irrelevant perceptual feature (spatial location), while the sequence of target identity and thus of responses was random. The RTs indicated that participants could learn the sequence even though they were instructed to focus on the cross. The authors concluded that sequence learning can occur without overt oculomotor movements. However, since the eyes were not recorded, it has been argued that saccades potentially occurred once the target (presented for $100 \mathrm{~ms}$ ) had already disappeared (Laubrock \& Kinder, 2014). Thus, the attested sequence learning might need to be attributed (at least partially) to oculomotor learning.

Conversely, different accounts (response learning, stimulus-response learning) suggest that a motor component is necessary in sequence learning (Koch \& Hoffmann, 2000; Willingham, 1999). Thus, both making a response and the location of stimulus/response are critical in sequential learning. For example, Willingham (1999) asked participants to perform the SRT task training phase by providing the corresponding manual responses with keypresses to a repeating spatial pattern (i.e., the push condition), or to simply watch the stimuli (i.e., the watch group). After the training phase, all participants went through a transfer phase, where they were asked to respond to the stimuli. There were two-random trial blocks and a sequenced block, followed by another random block. The results demonstrated faster RTs on the sequenced block for the push group compared to the watch group, supporting the notion that motor engagement is critical in sequence learning (although both groups responded in the transfer phase, making this a potential confounding factor). More recent studies have used oculomotor versions of the SRT 
PREDICTION AND IMPLICIT SEQUENCE LEARNING

task, where participants were instructed to follow the target on the screen with their eyes (Kinder, Rolfs, \& Klegl, 2008; Marcus, Karatekin, \& Markiewicz, 2006; Medimorec, Milin, \& Divjak, 2021; Vakil, Bloch, \& Cohen, 2017). While avoiding manual responses, the oculomotor versions of the SRT task do involve motor action, similar to the pattern observation version described above. The findings of the oculomotor SRT task studies usually closely resemble those found in the manual SRT task studies.

\section{Present Investigation}

In order to test between the accounts highlighting either sequence learning in the absence of any motor responses or the relative contribution of the motor processes to learning in the SRT task, it is necessary to find a manipulation that could restrict motor action while also allowing for a sequence to be perceived. To this end, we introduced an ocular version of the SRT task where participants' eye movements were restricted during the learning phase of the task. Specifically, participants were instructed to fixate on a marker, while the stimuli were presented paracentrally. While previous research has demonstrated that certain forms of learning, such as orientation discrimination and category acquisition can occur parafoveally (for a review, see Strasburger, Rentschler, \& Jüttner, 2011), we report the effects of such a manipulation in the context of sequential learning. It is important to note that the control of the manipulation was twofold: (1) participants' eye movements during the learning phase had to remain within the central interest area surrounding the fixation point, and (2) the target had to be visible during the learning phase. These points are further elaborated in the Procedure section. We then compared implicit learning in the restricted (fixated) SRT task (henceforth F-SRT) with implicit learning in the standard oculomotor SRT task (henceforth O-SRT; Kinder et al., 2008; Marcus et al., 2006). 
PREDICTION AND IMPLICIT SEQUENCE LEARNING

Thus, the design of the current study allows us to directly compare the effects of different variations of the SRT task (F-SRT vs. O-SRT) on learning of identically structured sequences. In the current study implicit learning was measured using anticipation measures, as they represent the strongest indicators of implicit sequence learning (Dale et al., 2012; Medimorec et al., 2021). We used two anticipation measures: the overall proportion of anticipations and the proportion of correct anticipations. While anticipations indicate learning strategies and are a precursor of sequence learning, correct anticipations indicate that the leaning actually took place. If if sequential learning does not necessarily rely on overt eye movements, we should expect no difference in learning between the two versions of the SRT task. Thus, the number of anticipations and the proportion of correct anticipations should be similar across the two tasks, with more correct anticipations in the learning block compared to the interference block. On the other hand, if sequence learning is a by-product of eye movements, there should be no learning in the restricted eye movement condition.

\section{Method}

\section{Participants}

Sixty-eight University students and staff ( 49 female; $M_{\text {age }}=27.5 ;$ no-SRT $=35$ ) participated in exchange for a $£ 7$ voucher. ${ }^{1}$ All participants had normal or corrected-to-normal vision. Four additional participants were excluded because they failed to complete the experiment.

\section{Design}

\footnotetext{
${ }^{1}$ The sample size per condition was selected to be close to a related recent experiment testing implicit sequence learning using oculomotor SRT tasks ( $n=30$; Vakil et al., 2017).
} 
PREDICTION AND IMPLICIT SEQUENCE LEARNING

The experiment used a 2-factor (O-SRT vs. F-SRT) between subject design. The dependent variables were the number of anticipations and the proportion of correct anticipations. Materials and Apparatus

Eye tracking was performed using the EyeLink Portable Duo eye tracker (SR research) paired with a 21 in. monitor (refresh rate: $60 \mathrm{~Hz}$; resolution: 1024 x 768 pixels). The eye tracker sampled at the rate of $500 \mathrm{~Hz}$ in the head stabilized mode. While viewing was binocular, tracking was monocular and used participant's dominant eye. The right eye was recorded for 48 participants $(71 \%)$.

SRT tasks. SRT tasks were implemented using OpenSesame (Mathôt, Schreij, \& Theeuwes, 2012). In the O-SRT task, stimuli consisted of five slides, each containing four white squares measuring 65 x $65 \mathrm{~mm}$, on a grey background (i.e., the white squares were interest areas; see Figure 1). A black circle (the target), $20 \mathrm{~mm}$ in diameter, appeared in the white squares in four slides. The fifth slide was the anticipatory slide without the target. The slides were presented $70 \mathrm{~cm}$ away from participants' eyes. The white squares subtended horizontal and vertical visual angles of $5.5^{\circ}$, and the target subtended visual angles of $1.7^{\circ}$. Our versions of the task contained six blocks. The learning sequence was presented across the first four blocks (i.e., learning phase, Blocks 1-4). Block 5 contained the interfering sequence, while the original learning sequence was repeated in Block 6.

In the F-SRT task, we used the same slides, only this time a fixation target was present in the center of the array throughout the first four blocks (i.e., learning blocks) on all slides. Since this condition required a relatively stable fixation for the extended period of time, we used a target which looks like a combination of bullseye and cross hair (see Figure 1). Previous research has demonstrated the effectiveness of this fixation target in minimizing involuntary eye 


\section{PREDICTION AND IMPLICIT SEQUENCE LEARNING}

movements (Thaler, Schütz, Goodale, \& Gegenfurtner, 2013). The fixation target diameter was $20 \mathrm{~mm}$, subtending visual angles of $1.7^{\circ}$. The surrounding central Interest Area (around the target) was a $72 \times 72 \mathrm{~mm}$ square, subtending visual angles of approximately $6^{\circ}$. The distance between the centers of the fixation target and the moving target within any of the squares was $68.5 \mathrm{~mm}$, corresponding to a visual angle of $5.8^{\circ}$. Thus, the moving target remained well within paracentral visual field (i.e., $<8^{\circ}$ ). Blocks 5 and 6 contained the counterbalanced interfering and learning sequences, otherwise identical to those in the O-SRT task.

\section{Figure 1}

Slides from the Experiment
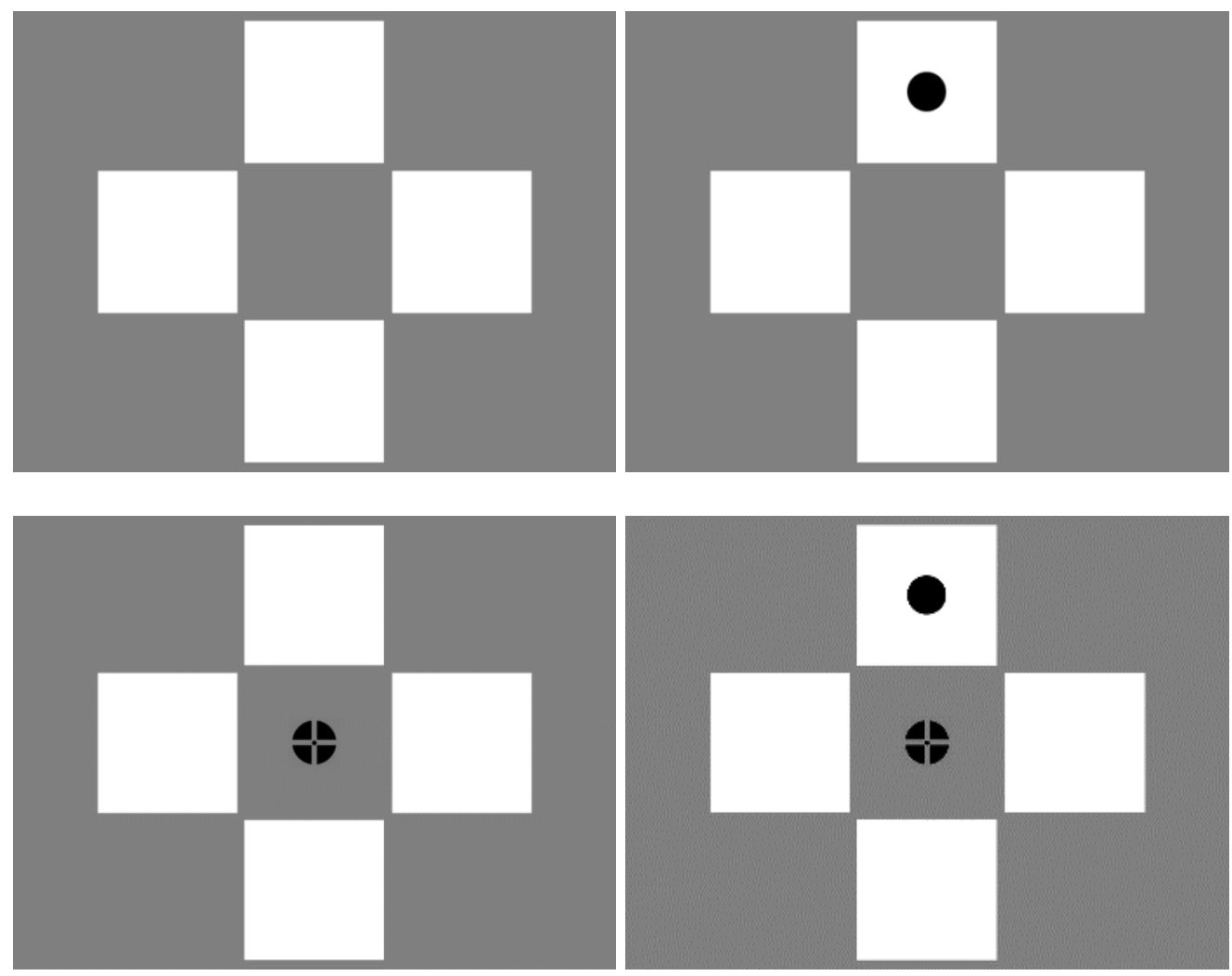


\section{PREDICTION AND IMPLICIT SEQUENCE LEARNING}

Note. Top slides are from the O-SRT task (left panel: anticipatory slide; right panel: a target slide). Bottom slides with the central fixation target are from the learning blocks of the F-SRT task.

Sequence structure. We used second-order conditional sequences (SOC; Vakil, et al., 2016; Wilkinson \& Shanks, 2004). SOC sequence learning is complex, because it requires second-order knowledge (i.e., a target location can be predicted only if the two preceding locations are considered). There were two sequences, “341243142132" and "342312143241", with the numbers 1-4 corresponding to four different positions (down, left, right, and up). Each sequence was either the learning or the interfering sequence, while the sequence order was counterbalanced across participants.

Anticipations and correct anticipations. Anticipations appeared when participants transitioned their gaze towards a different potential target location during the blank slide presentation (i.e., the first $500 \mathrm{~ms}$ of each trial). Anticipations were correct if the participant's gaze remained within the correct interest area at the time the target appeared (otherwise they were incorrect).

Explicit knowledge questionnaire. Participants were asked the following questions to assess sequence awareness: (1) Did you notice anything special about the experiment?, (2) Did you notice any patterns during the experiment?, (3) If yes, could you explicitly recall the pattern?, (4) If yes - please write the pattern down (this was a free recall question, and participants did not receive any additional information).

\section{Procedure}

Participants were randomly assigned to one of the conditions (O-SRT vs. F-SRT). At the beginning of each session, participants were calibrated using the 9-point calibration type. Drift 
PREDICTION AND IMPLICIT SEQUENCE LEARNING

correction was performed at the beginning of each block. The tasks began with twelve practice trials (randomly generated sequences; the instructions corresponded to a given condition, as described below).

In the O-SRT task, participants were instructed to follow the target on the screen with their eyes. The experiment consisted of six blocks, each containing a 12-element sequence repeated five times (i.e., 60 trials per block). At the beginning of each trial the anticipatory slide was presented for $500 \mathrm{~ms}$, followed by a presentation of the target for $1100 \mathrm{~ms}$. The first four blocks were learning blocks (Blocks 1-4). The recordings indicated that participants followed the target in $95.4 \%$ of trials. Each block started from a different point in the sequence. The learning blocks were followed by an interfering block, containing a different 12-element sequence (Block 5, i.e., interference). Finally, the original learning sequence was reintroduced in Block 6 (i.e., learning).

In the F-SRT task, participants were instructed to look at the fixation point at all times during the first four blocks (the learning blocks). Participants' eye movements were tracked to assure that they remained relatively stable, and trials were considered valid if the fixations remained contained within the central target fixation IA (i.e., the central square); this was true for $98 \%$ of the trials. Critically, the moving target was parafoveally visible to participants while they fixated on the central target (the was within paracentral visual field (i.e., $<8^{\circ}$ ) and target visibility was initially tested and confirmed in our pilot study; $n=8$ ). After the learning blocks, participants were instructed to start following the target with their eyes in the subsequent two blocks (5 and 6). Blocks 5 and 6 were interference and learning blocks containing the same slides as in the O-SRT task (here, the order of the two blocks was counterbalanced across participants). 
PREDICTION AND IMPLICIT SEQUENCE LEARNING

The task, administered in one session, took approximately 25 minutes to complete. After the SRT task, participants filled out the explicit knowledge questionnaire.

\section{Results}

We used R (R Core Team, 2019) and lme4 (version 1.1-21; Bates, Maechler, Bolker, \& Walker, 2015) to fit generalized linear mixed-effects models (GLMM binomial). We performed a series of mixed effects logistic regression analyses of anticipations and correct anticipations in the task (as our two DVs). The maximum model structure included the interaction between the SRT task (O-SRT vs. F-SRT) and block type (interference vs. learning). As a random effect we entered intercepts for participants. For each model, a fit to data was tested against a null model containing only the intercept. The models were compared using the anova function. Simple effects were calculated using the emmeans package (Lenth, 2021). The corresponding Bayesian analysis was performed using the brms package (Bürkner, 2017). ${ }^{2}$ Table 1 presents the overall number of anticipations and correct anticipations.

\footnotetext{
${ }^{2}$ All GLMM analyses were supplemented with Bayesian GLMMs (see Supplementary Materials). These analyses are provided to complement the frequentist analyses and to additionally confirm the strength of effects (when critical, analyses are presented in the text). Note that the estimates from both types of analyses were largely comparable.
} 
PREDICTION AND IMPLICIT SEQUENCE LEARNING

\section{Table 1}

The Number of Anticipations/Correct Anticipations across the Blocks and Conditions

\begin{tabular}{lcccc}
\hline & & \multicolumn{2}{c}{ Condition } & \\
\cline { 3 - 4 } Block & Anticipations & O-SRT & F-SRT & Total \\
\hline Interference & & 618 & 517 & 1135 \\
Learning & 673 & 543 & 1216 \\
Total & Correct Anticipations & & & \\
\hline \multirow{2}{*}{ Interference } & & 327 & 287 & 614 \\
Learning & & 389 & 280 & 669 \\
Total & & 716 & 567 & 1283 \\
\hline
\end{tabular}

\section{Anticipations}

For anticipations, the best-fit model did not include the interaction between the SRT task (O-SRT vs. F-SRT) and block type (interfere vs. learn). There was a main effect of block, estimate $=.11, S E=.05, z(8160)=2.09, p=.037$, such that the number of anticipations increased in the learning block compared to interference. There was no effect of task, estimate $=$ $-.19, S E=.20, z(8160)=-.98, p=.329$.

\section{Correct anticipations}

For correct anticipations, there was a significant interaction between the SRT task and block type, estimate $=-.38, S E=.17, z(2351)=-2.24, p=.025$ (see Figure 1$).$ In the simple effects analysis, in the O-SRT group accuracy increased in the learning block compared to the interference block, estimate $=.22, S E=.12, z=1.93, p=.054$. Using a Bayesian approach, this effect was statistically significant as indicated by the $95 \%$ credible interval, estimate $=.22,95 \%$ 
CI $[.003, .444]$. There was no difference in accuracy between the blocks in the F-SRT group, estimate $=-.16, S E=.13, z=-1.28, p=20$.

\section{Figure 2}

Predicted Values of Correct Anticipations Between the SRT tasks (Eye-Movement/O-SRT vs. No Eye-Movement/F-SRT)

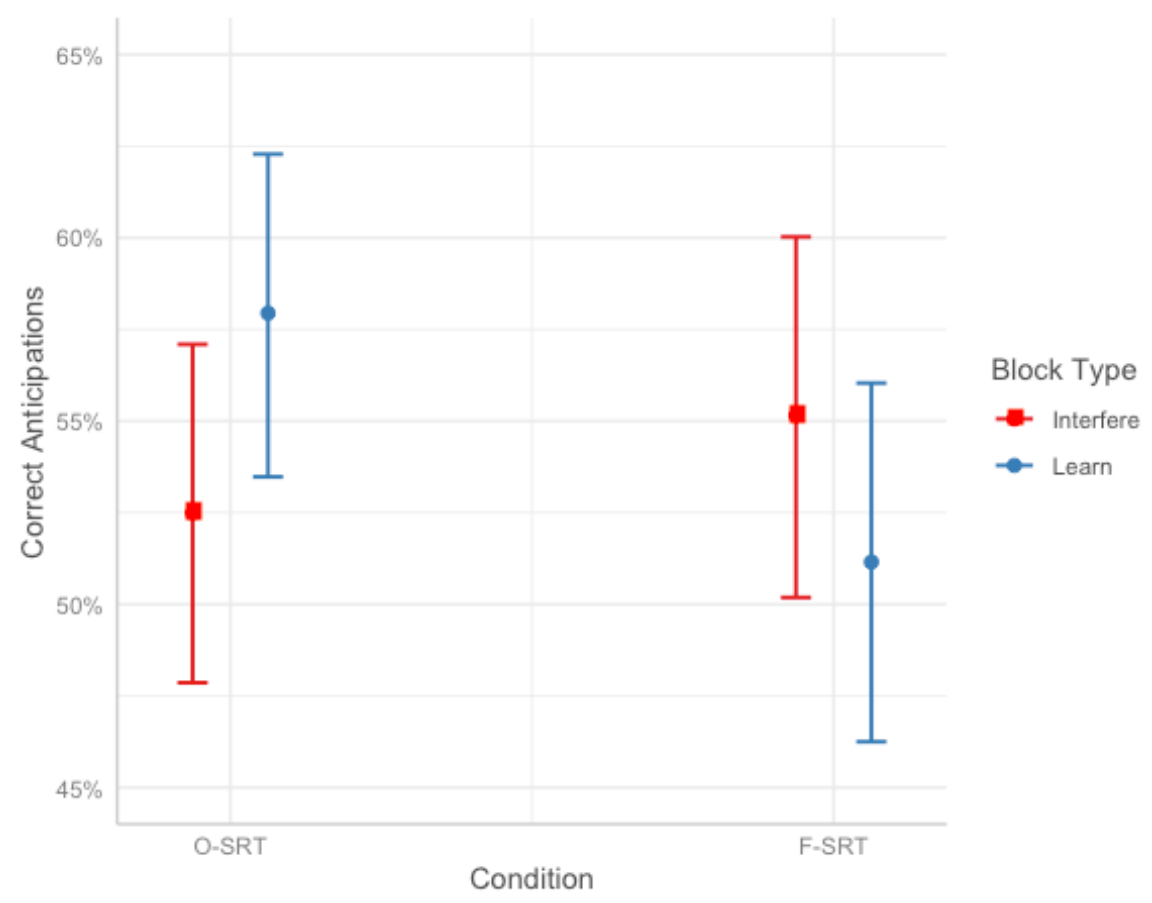

\section{Explicit Awareness}

Participants filled out the questionnaire to assess sequence awareness. Overall, $31 \%(n=$ $21, n \mathrm{O}-\mathrm{SRT}=11)$ of participants reported that they noticed something special about the experiment; $54 \%(n=37, n$ O-SRT $=17)$ reported that they noticed a pattern, while $18 \%(n=12$, $n \mathrm{O}$-SRT $=8)$ indicated that they could recall the pattern, and $16 \%$ of participants $(n=11, n$ O-SRT $=$ 8) attempted to generate the sequence. There were no differences between groups on these 


\section{PREDICTION AND IMPLICIT SEQUENCE LEARNING}

measures (all $\chi_{\mathrm{s}}^{2}<2.38, p_{\mathrm{s}}>.122$ ). The free recall test was scored following the standard procedure, where responses had to be part of a segment of at least three correct positions (see Willingham, 1999). Overall, 7 participants $($ nO-SRT $=5)$ produced correct strings ranging from 3 to $7(M=4.14, S D=1.68)$. These results suggest that although most participants reported that they detected some regularities in the task, very few acquired at least some explicit knowledge of the underlying 12-element trained sequence ${ }^{3}$.

\section{Discussion}

The current study investigated the mechanism underlying implicit sequence learning. We provided a test between different accounts of implicit sequence learning - one that highlights sequence learning in the absence of any motor responses and one that highlights the relative contribution of the motor processes to learning, by directly comparing the performance on the restricted and standard oculomotor sequence learning versions of the task. Moreover, we used different anticipation measures (anticipations and correct anticipations) as direct indicators of learning in these tasks.

A comparison at the group level provided evidence that sequence learning occurred in the standard version of the oculomotor SRT task (O-SRT). Correct anticipations in the learning block were found to increase in the O-SRT task relative to the interference block, while there was no difference between the blocks in the F-SRT task. This pattern indicates sequence learning in the O-SRT task, as the increase in correct anticipations when contrasting the interference block with the learning blocks signals sequence knowledge (Dale at al., 2012; Medimorec et al., 2021). Thus, one possible interpretation of the findings is that they support the notion that a

\footnotetext{
${ }^{3}$ Note that only participants who stated that they noticed a pattern and could explicitly recall it were asked to generate it. It is possible that other participants would also have been able to generate sequence portions indicating sequence knowledge.
} 


\section{PREDICTION AND IMPLICIT SEQUENCE LEARNING}

motor response is a requisite process in sequence learning. The lack of effect in the F-SRT condition could potentially indicate that, in the absence of any other motor responses, the explicit task to inhibit eye-movements actually interfered with learning of a spatial stimulus sequence. It is also worth noting at this point that the effect in the O-SRT task could likewise be interpreted to indicate the perceptual nature of sequence learning. If associations between the visual stimuli and the oculomotor responses are well established and can be considered relatively automatic, then there is no need to learn how to fixate visual targets (Kinder et al., 2008). However, such an interpretation assumes that visually fixating a target does not constitute a motor response, and this clearly differs from our operationalization of motor responses.

On the other hand, the results of our study also indicated that anticipations occurred more frequently in the learning block compared to the interference block regardless of the type of SRT task. Anticipations typically occur before knowledge indicated by correct anticipations (Dale at al., 2012; Medimorec et al., 2021). Specifically, anticipations seem to appear before correct anticipations emerge. Our results thus seem to suggest an interesting possibility that increased anticipations in the learning block in the no eye-movement task indicate a transitional period preceding the occurrence of correct anticipations. This would suggest that even individuals in the F-SRT group adopted a readiness to respond based on the presence of sequence regularity. Thus, learning seems to remain in earlier stages (or not yet consolidated) compared to learning in the O-SRT task. This conclusion is further supported by a relatively high correct anticipation rate in the F-SRT group (see Figure 1). There is evidence that participants in the standard oculomotor SRT task begin responding with above chance precision relatively early in the task, sometimes as soon as the first block (Tal \& Vakil, 2020; Vakil, Ashkenazi, Nevet-Perez, \& Hassin-Baer, 2021). Interestingly, recent studies have also reported relatively high precision even in the 
PREDICTION AND IMPLICIT SEQUENCE LEARNING

interference block, suggesting a surprisingly rapid adaptation to the new sequence (Tal \& Vakil, 2020). It has been suggested that the latter phenomenon may indicate statistical learning achieved previously in the task, since individuals continue to be sensitive to the task grammar or the presence of sequence regularity (Tal \& Vakil, 2020). While this does not necessarily indicate learning of the new interference sequence, it does suggest a behavioral adaptation driven by sensitivity to the rules governing the previously presented learning sequence structure. Relatively high anticipation rates in both blocks in the F-SRT group could indicate that such sensitivity to sequence regularity had occurred, while there was still no evidence of learning of the original sequence (which could potentially explain a surprising albeit not statistically significant trend in correct anticipations between the blocks in this group $)^{4}$. Thus, as discussed previously, it is possible that, in the absence of motor responses, the explicit task to inhibit oculomotor responses disrupted learning in the F-SRT condition.

Finally, several questions remain unresolved and these pertain to the mechanism underlying learning in the SRT task in general and in relation to pure perceptual learning. For example, it is possible that the underlying learning mechanism varies with the type of the to-belearned perceptual features, such as perceptual modality (e.g., visual vs. auditory, Koch, Blotenberg, Fedosejew, \& Stephan, 2020). Another interesting question is how working memory capacity interacts with different types of learning potentially underlying the SRT task

(Medimorec et al., 2021)

\section{Conclusion}

The current study provided a test between the accounts highlighting either sequence learning in the absence of motor responses or the relative contribution of the motor processes to

\footnotetext{
${ }^{4}$ The trend was not driven by block order.
} 
PREDICTION AND IMPLICIT SEQUENCE LEARNING

learning. The results were consistent with the former account: eye movements appear to be a prerequisite for sequence learning. However, a change in anticipatory behavior in the restricted movement task indicated the interesting possibility that implicit learning can happen even when eye-movements are suppressed, if a sufficient amount of exposure is provided.

\section{Declarations}

Conflicts of interest: The authors declare that they have no conflict of interest.

Ethics approval: All procedures performed in studies involving human participants were in accordance with the ethical standards of the University's Ethics Committee and with the 1964 Helsinki declaration and its later amendments or comparable ethical standards. The data were collected as part of the Out of Our Minds project.

Consent to participate: Informed consent was obtained from all individual participants included in the study.

Open Practices Statement: The data set supporting the conclusions of this article is available as an electronic supplementary material and at https://doi.org/10.25500/edata.bham.00000697 The $\mathrm{R}$ code necessary to reproduce our models is available at https://github.com/ooominds/Inhibition-of-eye-movements-disrupts-spatial-sequence-learning

Note: This version of the article may not completely replicate the final authoritative version published in Experimental Psychology. It is not the version of record and is therefore not suitable for citation. Please do not copy or cite without the permission of the authors. 
PREDICTION AND IMPLICIT SEQUENCE LEARNING

\section{References}

Bates, D., Maechler, M., Bolker, B., Walker, S., (2015). Fitting Linear Mixed-Effects Models Using lme4. Journal of Statistical Software, 67, 1-48.

Bürkner, P. C. (2017). brms: An R package for Bayesian multilevel models using Stan. Journal of Statistical Software, 80, 1-28.

Cleeremans, A. \& McClelland, J. L. (1991). Learning the structure of event sequences. Journal of Experimental Psychology: General, 120, 235-253.

Cohen, A., Ivry, R. \& Keele, S. W. (1990). Attention and structure in sequence learning. Journal of Experimental Psychology: Learning, Memory, and Cognition, 16, 17-30.

Coomans, D., Deroost, N., Vandenbossche, J., Van den Bussche, E., \& Soetens, E. (2012). Visuospatial perceptual sequence learning and eye movements. Experimental Psychology, 39, 279-285.

Dale, R., Duran, N. D., \& Morehead, J. R. (2012). Prediction during statistical learning, and implications for the implicit/explicit divide. Advances in Cognitive Psychology, 8, 196209.

Haider, H., Eberhardt, K., Kunde, A., \& Rose, M. (2013). Implicit visual learning and the expression of learning. Consciousness and Cognition, 22, 82-98.

Howard, J. H., Mutter, S. A. \& Howard, D. V. (1992). Serial pattern learning by event observation. Journal of Experimental Psychology: Learning, Memory, and Cognition, 18, 1029-1039.

Kinder, A., Rolfs, M., \& Kliegl, R. (2008). Sequence learning at optimal stimulus-response mapping: Evidence from a serial reaction time task. Quarterly Journal of Experimental Psychology, 61, 203-209. 


\section{PREDICTION AND IMPLICIT SEQUENCE LEARNING}

Koch, I., \& Hoffmann, J. (2000). The role of stimulus-based and response-based spatial information in sequence learning. Journal of Experimental Psychology: Learning, Memory, \& Cognition, 26, 863-882.

Koch, I., Blotenberg, I., Fedosejew, V., \& Stephan, D. N. (2020). Implicit perceptual learning of visual-auditory modality sequences. Acta Psychologica, 202, 102979.

Laubrock, J., \& Kinder, A. (2014). Incidental sequence learning in a motion coherence discrimination task: How response learning affects perception. Journal of Experimental Psychology: Human Perception and Performance 40, 1963-1977.

Lenth, R.V. (2021). emmeans: Estimated Marginal Means, aka Least-Squares Means. R package version 1.6.0. https://CRAN.R-project.org/package=emmeans

Marcus, D. J., Karatekin, C., \& Markiewicz, S. (2006). Oculomotor evidence of sequence learning on the serial reaction time task. Memory \& Cognition, 34, 420-432.

Mathôt, S., Schreij, D., \& Theeuwes, J. (2012). OpenSesame: An open-source, graphical experiment builder for the social sciences. Behavior Research Methods, 44, 314-324.

Medimorec, S., Milin, P. \& Divjak, D. (2021). Working memory affects anticipatory behavior during implicit pattern learning. Psychological Research, 85, 291-301.

Nissen, M. J., \& Bullemer, P. (1987). Attentional requirements of learning: Evidence from performance measures. Cognitive Psychology, 19, 1-32.

R Core Team (2018). R: A language and environment for statistical computing. R Foundation for Statistical Computing, Vienna, Austria. URL https://www.R-project.org/.

Remillard, G. (2011). Pure perceptual-based learning of second-, third-, and fourth-order sequential probabilities. Psychological Research, 75, 307-323. 


\section{PREDICTION AND IMPLICIT SEQUENCE LEARNING}

Saffran, J. R., Aslin, R. N., \& Newport, E. L. (1996). Statistical learning by 8-month-old infants. Science, 274, 1926-1928.

Schwarb, H., \& Schumacher, E. H. (2012). Generalized lessons about sequence learning from the study of the serial reaction time task. Advances in Cognitive Psychology, 8, 165-178.

Strasburger, H., Rentschler, I., \& Jüttner, M. (2011). Peripheral vision and pattern recognition: A review. Journal of Vision, 11, 13.

Tal, A., \& Vakil, E. (2020). How sequence learning unfolds: Insights from anticipatory eye movements. Cognition, 201, 104291.

Thaler, L., Schtz, A. C., Goodale, M. A., \& Gegenfurtner, K. R. (2013). What is the best fixation target? The effect of target shape on stability of fixational eye movements. Vision Research, 76, 31-42.

Turk-Browne, N. B, Scholl, B. J., Chun, M. M., Johnson, M. K. (2009). Neural evidence of statistical learning: Efficient detection of visual regularities without awareness. Journal of Cognitive Neuroscience, 21, 1934-1945.

Vakil, E., Ashkenazi, S. S., Nevet-Perez, M., \& Hassin-Baer, S. (2021). Implicit sequence learning in individuals with Parkinson's disease: The added value of using an ocular version of the serial reaction time (O-SRT) task. Brain and Cognition, 147, 105654.

Vakil, E., Bloch, A., \& Cohen, H. (2017). Anticipation measures of sequence learning: Manual versus coulometer versions of the serial reaction time task. Quarterly Journal of Experimental Psychology, 70, 579-589.

Wilkinson, L., \& Shanks, D. R. (2004). Intentional control and implicit sequence learning. Journal of Experimental Psychology: Learning, Memory, \& Cognition, 30, 354-369. 


\section{PREDICTION AND IMPLICIT SEQUENCE LEARNING}

Willingham, D. B. (1999). Implicit motor sequence learning is not purely perceptual. Memory \& Cognition, 27, 561-572. 